\title{
Fuzzy Approach to the Selection of Material Data in Concurrent Engineering Environment
}

\author{
Adavi Balakrishna ${ }^{1}$, G. Ravi Chandra ${ }^{2}$, Balakrishna Gogulamudi ${ }^{3}$, Chinta Someswararao ${ }^{4}$ \\ ${ }^{1}$ Department of Mechanical Engineering, SRKR Engg College, Bhimavaram, India \\ ${ }^{2}$ Department of Mechanical Engineering, Vardhaman College \\ of Engineering, Hyderabad, India \\ ${ }^{3}$ Department of Mechanical Engineering, PVP Siddartha Institute \\ of Technology, Vijayawada, India \\ ${ }^{4}$ Department of CSE, SRKR Engg College, Bhimavaram, India \\ E-mail: chinta.someswararao@gmail.com
}

Received June 27, 2011; revised August 24, 2011; accepted September 2, 2011

\begin{abstract}
The selection of proper materials for a structural component is critical in engineering design. Existing design procedures may currently be sufficient, especially where experience exists, but fierce industrial competition is spurring the search for improved methods and tools. The main drivers are quality, life-cycle cost, and time-to-market. Improved design efficiency and accuracy may have an enormous impact on the economic viability of the final product. The use of computer-aided systems can assist the designer in selecting the apropriate material these may potentially reduce product cost and time-to-market while assisting the concurent engineering activities, thereby resolving problems related to materials presented during the initial phase of design. This paper focuses on specific issues such as material selection at early design stage. In this paper material selection algorithm is developed using fuzzy logic technique for selection of proper material from database as per design engineers' criteria. The information obtained from the selection algorithm is exchanged through a properly secured web page through pure internet communication to different users in the enterprise so that it can create concurrent engineering environment throughout the product life cycle.
\end{abstract}

Keywords: Material Properties, Concurrent Engineering, Fuzzy Logic and Material I.D., Web Pages, CPD

\section{Introduction}

In the Concurrent Engineering (CE) environment, techniques, algorithms and software tools are provided to allow designers and developers to interact with each other effectively and efficiently. Implementation of $\mathrm{CE}$, would naturally consume more time and money at the initial design stage so as to ensure that the concept selection is optimized, companies can reduce design changes at a later stage, leading to better engineered products with an advantage in total quality, time and cost competitiveness.

Material selection is one of the most important activeties for a product development process. In the modern design and manufacturing environment, such as, newly developed CE methodology, material selection plays as important role as other activities in the total design model such as market investigation, product design specifica- tion, component design, design analysis, manu- facture and assembly is shown in Figure 1 [1]. Material selection is one of the CE strategies. The integrated ma- terial selection system thus plays a major role in product development to optimize the cost of product.

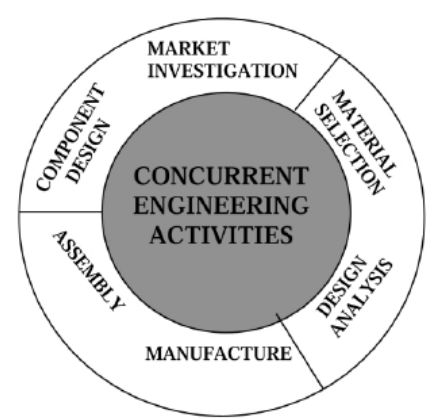

Figure 1. The importance of material selection in product development 
The selection of the most suitable material for particular application is crucial function in the design of an artifact. The performance of materials is important in terms of service conditions, processing and its economic, the availability of candidate materials, the company's make or buy decision for the system components; the customer preferences; and most importantly, the total life-cycle cost. In practice, it may be necessary to express the performance in terms of fuzzy numbers. The design engineer is responsible for selecting relevant properties and assessing the importance of the selected properties based on the requirements of a particular application.

This paper discusses one of the CE integration strategies i.e., 'selection of material' using Fuzzy Logic that can be adapted for successful integration within industry and beyond using web services. Each material in the database is an alternative to be selected for the application. A computerized system, which provides access to material data, is not necessarily a material selection system, although access to data is essential to facilitate selection. The material selection system assess the suitability of all materials in the database, eliminates unsuitable materials from further consideration, and gives a final ranking to all of the suitable materials.

\section{Review of CE Environment and Material Selection Systems}

The CE philosophy can be implemented through different strategies considering the diverse requirements of users and the conditions of the companies. Pallot and V. Sandoval [2] emphasized the communication and sharing of design data, integration and interoperability of the application systems in design and manufacturing, and the coordination between the upstream and downstream activities to support concurrent activities for multi-disciplinary teams. According to Prasad [3], materials are the common elements enumerating parts in a product realization process. Normally, design engineers rely on the materials that they are familiar with. However, when the design requirements exceed the constraints of such materials or exceed the constraints on material properties, $\mathrm{CE}$ team members must consider alternative materials. With direct online access to a materials database, the CE team members could select materials that are lighter, stronger and lower in cost.

Within the engineering design and manufacturing context, there have been many research works for the integration of various engineering data, models or software tools, besides the SHAred Dependency Engineering project (SHADE), Component Agent Design-Oriented Model (CADOM), Responsible Agents for ProductProcess Integrated Design (RAPPID), Knowledge Inten- sive Engineering Framework (KIEF) and Karlsruhe's Virtual Documentation (KaViDo). These are only the beginning and more work remains to be done in this direction [4]. Besides all these technologies, information exchange systems supporting distributed engineering, CE and Collaborative Product Development (CPD) are being reviewed in this paper.

Masaharu Yoshioka [5] discussed Knowledge Intensive Engineering Framework (KIEF) tool for supporting various kinds of engineering tools that can be integrated by intensively storing engineering knowledge base and representing the relationship among them. Karlsruhe's Virtual Documentation, a web-based system tool for collaborative research and development, is presented by Oliver Tamine and Rudiger Dillmann [6]. It ensures that product information and construction knowledge are shared between the project collaborators. A CPD System boundary [7] developed under the DST project at our institute is used in the present paper. It integrates designer, analyzer and manufacturer, as clients to the main server in an extended enterprise using web page so that it creates a CE environment. White [8] emphasised the importance of computer aided materials selection over manual selection of materials from hand books. S.M. Sapuan [9] discussed the importance of Knowledge Based System (KBS) in the context of concurrent engineering and studied KBS in material selection in an en-gineering design process.

$\mathrm{CE}$ design requires simultaneous evaluation and material selection of several design attributes, including manufacturing costs, weights, quality, and mechanical design considerations. The interrelationships between these factors have not been considered in the design process until recently. Dong-Hyun jee and Ki-Ju Kang [10] proposed a method for optimal material selection using TOPSIS to rank the candidate of materials. A manual procedure is adopted for ranking.

The values of material properties are often qualitatively described or imprecisely measured using ranges. Material properties are also of varying degrees of importance for different design requirements. The desired value and importance of weights of material properties are usually described in a linguistic fashion. It is not easy to precisely quantify the rating of each alternative mate- rial. This impreciseness in the material selection process motivates us to develop a multi criteria decision-making method using fuzzy set theory. Fuzzy set theory [11] was developed exactly based on the premise that the key elements in human thinking are not numbers, but linguis tic terms or labels of fuzzy sets.

Knosala and Pedrycz [12] described a fuzzy multi objective decision-making method dedicated to the evaluation of design alternatives in mechanical engineering. 
Singer [13] described a fuzzy decision method for planning the maintenance of urban infrastructural systems consisting of a large number of subsystems when the resources available are inadequate. Maeda and Murakami [14] proposed a fuzzy decision-making method for a multiple-objective problem to decide what kind of computer system could be acquired for research and education in a computer engineering department.

Cebon and Ashby $[15,16]$ developed a computerized materials selection system called Cambridge Materials Selector. The system uses materials selection charts, which are displaying material property data through the use of fuzzy technique.

Materials data supports many different functions in manufacturing organizations from the initial phases of design to the disposal requirements at the end of a products life cycle [17]. The most critical of these functions is the process of analysis used to predict the products' performance and its end use in the environment.

The introduction of computerized design and analysis systems offered the promise of selecting the right material for an optimized design early in the product development cycle. This paper proposes a material selection system to select required material from database using fuzzy logic technique and exchange data in CE environment. This work is one of the supporting tools to the existing CPD system developed under the DST project at our institute.

\section{Modelling of Material Selection Using Fuzzy Logic}

The developments of fuzzy decision making theories have greatly contributed to their gradually increased applications including material selection.

\subsection{Fuzzy Material Properties}

Physical properties include density, specific heat, thermal expansion and conductivity, melting point, and electrical and magnetic properties. Important mechanical properties are strength, toughness, hardness, elasticity, fatigue, creep, and strength-to-weight ratio. These material properties can be generally classified into the following two categories based on the way they are measured:

1) Qualitative Properties: Some material properties cannot be easily quantified, but instead are qualitatively described in fuzzy terms such as "recommended," "acceptable," "not recommended," or other terms such as "excellent," "good," "fair," "poor," and so on. One example of such a property is corrosion resistance.

2) Quantitative Properties: Such properties can be ex- pressed numerically. Due to the stochastic nature of material processing operations, such properties are usually not fixed, but range between two values (minimum and maximum). For example, the hardness of stainless steel is approximately $(270,420)$ HB. Such quantitative properties are represented as fuzzy numbers of type 1 . Sometimes, the minimum and maximum for the range interval are not certain either. Such quantitative properties can be considered as fuzzy numbers of type 2 . The present study confined to fuzzy numbers of type 1 .

\subsection{Suitability of a Material}

The database used in this work has six different materials from which a relevant material can be retrieved, for different cases, based on the proposed methodology. A systematic approach to the material selection problem by using the concept of fuzzy set theory is proposed. The procedure for material selection to find the ranked list of materials from the database is explained in flow chart shown Figure 2.

A fuzzy suitability index is proposed to indicate the suitability of a material for an engineering design application[18]. The index accounts for the ambiguities involved in the evaluation of the appropriateness of alternate materials and the importance of material properties. Liou and Wang [19] are proposed a method of ranking fuzzy numbers with integral value is used in this paper for ranking the suitable materials.

\section{Framework of the Program}

C language is employed in present work for generalizing the material selection problem. The menu driven user interactive version is quite flexible in obtaining outputs of complex material properties. The input parameters used in this program are design engineer criteria (desired material property values). The main objective of the program is to generate an output displaying the selected materials with ranks, along with the number of combinations of criteria such as high and low. This information can be useful for the designer to check the suitability of the selected material as per his choice, there by he (designer) can resolve concurrency in the material selection. The following steps explain fuzzy logic procedure.

Step-1: The membership functions can be formed by graphical representations. The graphical representations may include different shapes like triangle, trapezoidal, Gaussian, etc. The trapezoidal membership function is considered here.

The desired material property values (specified by designer) are converted into trapezoidal fuzzy numbers. Let $D_{j}=\left(d a_{j}, d b_{j}, d c_{j}, d d_{j}\right)$ be the desired material property 


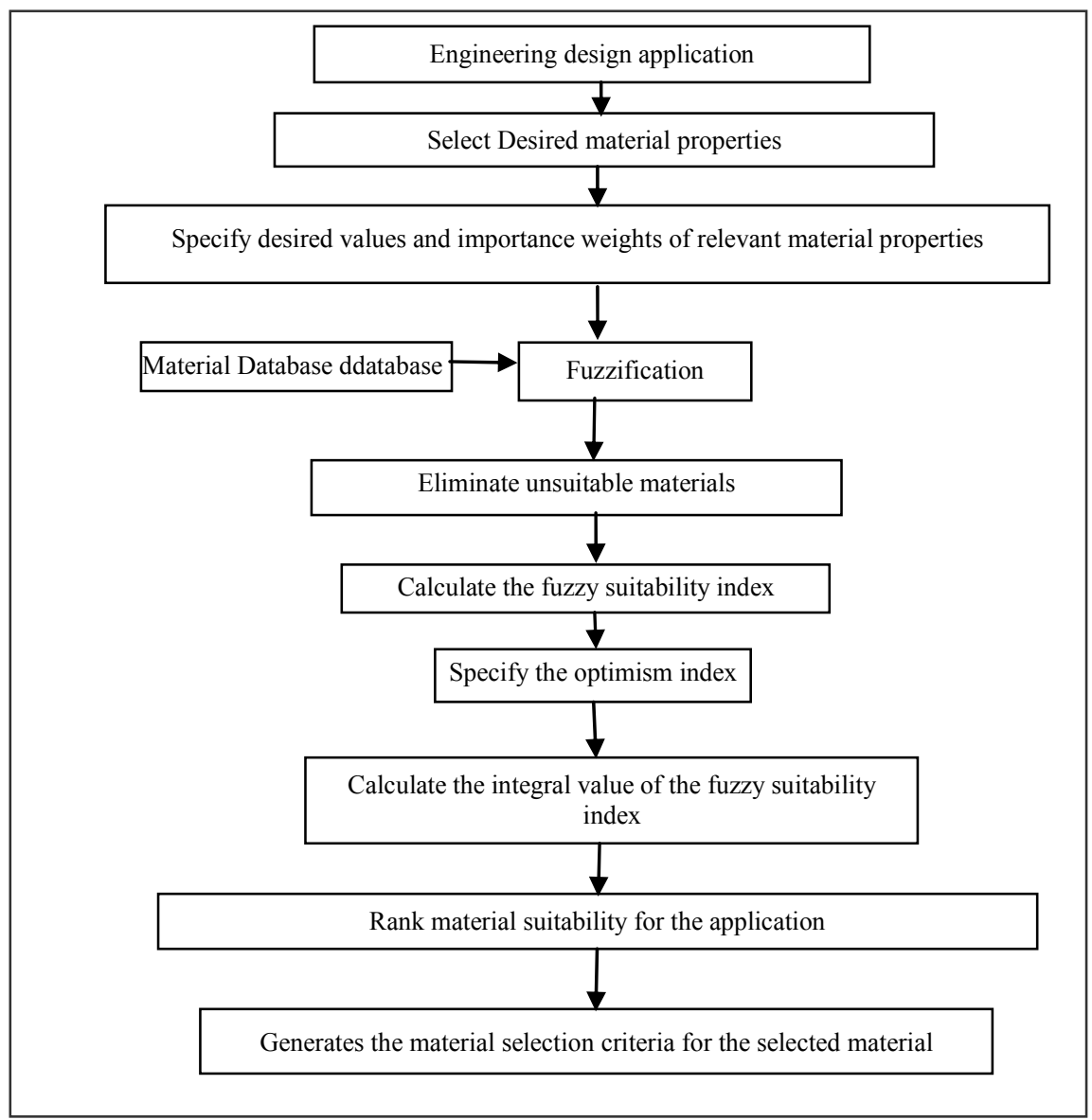

Figure 2. Flow chart of material selection based on fuzzy set theory

values and $P_{i j}=\left(p a_{i j}, p b_{i j}, p c_{i j}, p d_{i j}\right)$ be the property value of property $j$. The value of $D_{j}$ is usually specified in terms of a rough number or a range by the design engineer. Once the value is specified, the system automatically converts it into a trapezoidal fuzzy number. Let a desired value of the relevant property be $270\left(D_{1}\right)$, so that the corresponding trapezoidal fuzzy numbers is given by $D_{1}$ $=(243,270,270,300)$.

Step-2: The important weights of material properties are transformed into trapezoidal fuzzy numbers. The importance of a particular property in material property set can be determined by assigning weight to that property. Design engineer assigns a sample of weights to desired properties based on the design criteria. For example $\{L, M, H\}$ meaning "Low", "medium", and "high", to evaluate the importance of a material property. The importance of weight is a linguistic variable with the set of possible values subjectively determined using equations (1) to (6). The upper bound and lower bound are set to be 0 and 1 as per convention. The important weights can be assessed by requesting the design engineer to weigh the property through the designed rating scale. L: $(0,0.3,0.3$, $0.5)$

$$
\begin{aligned}
& f(x)=1-10 x / 3 \quad 0 \leq x \leq 0.3 \\
& =5 / 2-5 x \quad 0.3 \leq x \leq 0.5 \\
& M:(0.2,0.5,0.5,0.8) \\
& f(x)=10 x / 3-2 / 3 \quad 0.2 \leq x \leq 0.5 \\
& =8 / 3-10 x / 3 \quad 0.6 \leq x \leq 0.8 \\
& H:(0.5,0.7,0.7,1) \\
& f(x)=5 x-5 / 2 \quad 0.5 \leq x \leq 0.7 \\
& =10 / 3-10 x / 3 \quad 0.7 \leq x \leq 1
\end{aligned}
$$

Regardless of which approach is used, the importance of weights need to be represented as trapezoidal fuzzy numbers, denoted by $w_{j}=\left(w a_{j}, w b_{j}, w c_{j}, w d_{j}\right)$, before they can be used for assessing the suitability of a material.

Step-3: The values of the relevant material properties in the database are converted into trapezoidal fuzzy numbers as explained in the step- 1 .

Step-4: All of the unsuitable materials are identified and eliminated from further consideration using rule: 
Material ' $i$ ' is concluded to be unsuitable if $p d_{i j} \leq \mathrm{da}_{\mathrm{j}}$ whenever a large value of property $j$ is desired or $p a_{i j} \geq$ $d d_{j}$ whenever a small value of property $j$ is desired.

Step-5: Material rating is calculated and assigned as $S_{i j}$ to material ' $i$ ' for property ' $j$ '. $S_{i j}$ is evaluated based on the compatibility between the adjusted material property $A P_{i j}$ and the desired property $D_{j}$ for the application. The value of $D_{j}$ is usually specified in terms of a rough number or a range by the design engineer. Once the value is specified, the system automatically converts it into a trapezoidal fuzzy number as discussed in step 1. $S_{i j}$ value calculated using equations from (7) to (12) for given $P_{i j}$ and large value of $D_{j}$ is desired. $S_{i j}$ is a trapezoidal fuzzy number and is denoted by $\left(s a_{i j}, s b_{i j}, s c_{i j}, s d_{i j}\right)$.

$$
S_{i j}=\left(A P_{i j} \Theta D_{j}\right) \div D_{j}
$$

where

$$
A P_{i j}-n d_{j} \oplus\left(p a_{i j}, p b_{i j}, p c_{i j}, p d_{i j}\right)
$$

and

$$
n d_{j}=\left|\min \left(p a_{i j}\right)-d d_{j}\right|
$$

On the other hand it is desired to calculate $S_{i j}$ value using equations from (10) to (12) for given $P_{i j}$ and smaller value of $D_{j}$.

$$
S_{i j}=\left(D_{j} \Theta A P_{i j}\right) \div D_{j}
$$

where

$$
A P_{i j}=\left(p a_{i j}, p b_{i j}, p c_{i j}, p d_{i j}\right) \Theta n d_{j}
$$

and

$$
n d_{j}=\left|d a_{j}-\max \left(p d_{i j}\right)\right|
$$

Step-6: Final fuzzy suitability index is computed for material $S_{i}$ from the set of materials ' $i$ ' using Equation 13.

$$
S_{i}=1 / n \otimes\left[\left(S_{i 1} \otimes w_{1}\right)+\left(S_{i 2} \otimes w_{2}\right) \oplus \cdots \oplus\left(S_{i n} \otimes w_{n}\right)\right](13)
$$

where $n$ is the total number of relevant material properties selected for the engineering design application.

Step-7: Material rank is computed using total integral values. If ' $\mathrm{A}$ ' is a fuzzy number with membership function $f_{A}(x)$, then the total integral value with index of optimism $\mu$ is defined as Equation (14).

$$
I_{T}^{\mu}=\mu I_{R}(A)+(1-\mu) I_{L}(A)
$$

where $I_{R}(A)$ and $I_{L}(A)$ are the right and left integral values of $A$ respectively, and $\mu=[0,1] . I_{L}(A)$ and $I_{R}(A)$ are defined as

$$
I_{L}(A)=\int_{0}^{1} g_{A}^{L}(y) \mathrm{d} y
$$

$$
I_{R}(A)=\int_{0}^{1} g_{A}^{R}(y) \mathrm{d} y
$$

where $g_{A}^{L}(y)$ and $g_{A}^{R}(y)$ are the inverse functions of $f_{A}^{L}(y)$ and $f_{A}^{R}(y)$, respectively. The index of optimism $(\mu)$ represents the degree of optimism of a decision maker. The larger value of $\mu$ indicates a higher degree of optimism.

The inverse functions of $f_{A}^{L}(y)$ and $f_{A}^{R}(y)$ for trapezoidal fuzzy numbers are $g_{A}^{L}(y)=\alpha+(\beta-\alpha) y$ and $g_{A}^{R}(y)=\delta+(\tau-\delta) y$, respectively. Thus

$$
\begin{gathered}
I_{L}(A)=\int_{0}^{1} g_{A}^{L}(y) \mathrm{d} y=\int_{0}^{1}[\alpha+(\beta-\alpha) y]=\mathrm{d} y \frac{\alpha+\beta}{2} \\
I_{R}(A)=\int_{0}^{1} g_{A}^{R}(y) \mathrm{d} y=\int_{0}^{1}[\delta+(\tau-\delta) y] \mathrm{d} y=\frac{\tau-\delta}{2}
\end{gathered}
$$

Given the value $\mu$ (the design engineer decides it), the total integral value of the trapezoidal fuzzy number $A$ can be directly obtained as:

$$
I_{T}^{\mu}(A)=\frac{1}{2}[\mu(\tau+\delta)+(1-\mu)(\alpha+\beta)]
$$

The ranking of material 1 is said to be higher than material 2 if $I_{T}^{\mu}\left(S_{1}\right)>I_{T}^{\mu}\left(S_{2}\right)$. The total integral value of the trapezoidal fuzzy number ' $A$ ' can be calculated for different values $\mu$, i.e. $\mu=1,0, \& 0.5$, using Equation (19).

C-Program for fuzzy logic approach is being developed in the present work. Execution of this program generates an output displaying the selected materials with ranks, along with the number of combinations of criteria such as high and low. This information can be useful for the designer to check the suitability of the se- lected material as per engineer's choice there by resolv- ing concurrency in the material selection.

\section{Case Study}

Fuzzy logic approach developed in this paper is tested for selection of material for propulsor. The small database is considered, after initial screening material database, because to represent property value in graphically for validating results. The results are presented here on propulsor because small data and also validate result graphically also.

\section{Problem Description of Propulsor}

Ships and Under-water vehicles like Torpedoes, Submarines, Submersibles, etc., are used in defense applications. These weapons require propulsor to drive the vehicle. The performance of the vehicle depends on the efficiency of the propulsor and its material. Propulsor is de signed for moderate and extreme depth and requires minimization of structural weight for increasing payload 
Table 1. Material data of aluminum alloys.

\begin{tabular}{|c|c|c|c|c|c|c|c|}
\hline S.No & $\begin{array}{l}\text { Name of the } \\
\text { Al-alloy }\end{array}$ & $\begin{array}{l}\text { Tensile strength } \\
\text { (MPa) }\end{array}$ & Brinell hardness & Strength/Weight & $\%$ of elongation & $\begin{array}{l}\text { Melting Point } \\
\left.\text { ( }{ }^{\circ} \mathrm{C}\right)\end{array}$ & $\begin{array}{l}\text { Proof Stress } \\
\text { (MPa) }\end{array}$ \\
\hline 1 & Al-24345 & 280 & 105 & 103.7 & 2 & 650 & 230 \\
\hline 2 & Al-6061 & 310 & 95 & 114.81 & 17 & 580 & 276 \\
\hline 3 & Al-6009 & 230 & 62 & 85.185 & 25 & 619 & 125 \\
\hline 4 & Al-5083 & 330 & 84 & 124.5 & 17 & 570 & 240 \\
\hline 5 & Al-5251 & 210 & 58 & 78.06 & 14 & 625 & 165 \\
\hline 6 & Al-6262 & 360 & 120 & 132.8 & 3 & 582 & 330 \\
\hline
\end{tabular}

performance/speed and operating range. Hence, the material used for propulsor must be light, strong, and ductile, easy to cast and machine, resistant to corrosion. Ship propulsor may be manufactured from commercially available materials like gray cast iron, low carbon steels, chromium-stain less steel, chromium-nickel, austenitic stainless steel, manganese bronze, nickel-manganese bronze, nickel-aluminum bronze, naval brass, and forged aluminum.

In the past design engineers were selecting the material by experience, on the basis of strength. The list of currently available materials is so lengthy that it even challenges the ability of the materials' engineer to make the most effective material selection. A user interactive object oriented program is developed to determine the material I.D, which satisfies design criteria and designer requirement.

Aluminum alloys are taken for the application purpose because of its vital role in the marine application, since the corrosion resistance and the life is long, the weight is light, price is low, and having more strength. Different series and quantitative properties of Aluminum alloy are presented in Table 1.

\section{Results}

The designer enters input data based on the experience. Design Engineer specifies choices of material properties, quantitatively or qualitatively, through Material Seletion Webpage. Let the designer select criteria for above said case such as $<$ tensile strength $>300 \mathrm{MPa},<$ hardness $>100$ BHN, <Strength/Weight value $>100,<$ percentage of elongation $>1,<$ melting point $>580^{\circ} \mathrm{C},<$ proof stress $>$ $220 \mathrm{MPa}$. The fuzzy logic program generates output of Al-24345. Thus designer entered data corresponds to the alloy with ID of Al-24345.

The above case is verified graphically. A graph is drawn by taking the property number on horizontal axis and normalized property values on vertical axis. Property numbers 1 to 6 are used in graph instead of properties such as tensile strength, hardness, Strength/Weight value, percentage of elongation, melting point and proof stress respectively. The input data entered by designer coincides with the required material i.e., Al-24345 as shown in Figure 3. In the graph, the legend 1 represents Al-24345 and legend 7 represents input data.

\section{Conclusions}

A fuzzy approached is proposed to support the material selection decisions in $\mathrm{CE}$ environment. A quantitative relationship between mechanical properties of aluminum alloys is presented through the fuzzy logic methodology. This method is extendable to any design application. The implementation of the methodology can be used to integrate material database with designer criteria and also assist designer to select material automated for intended application. This provides material data in machine readable electronic format to clients such as analysis engineers and manufacturing engineers in the CPD system. The weights are fixed

\section{Acknowledgements}

This work is partially supported by Department of Science \& Technology, Government of India, New Delhi

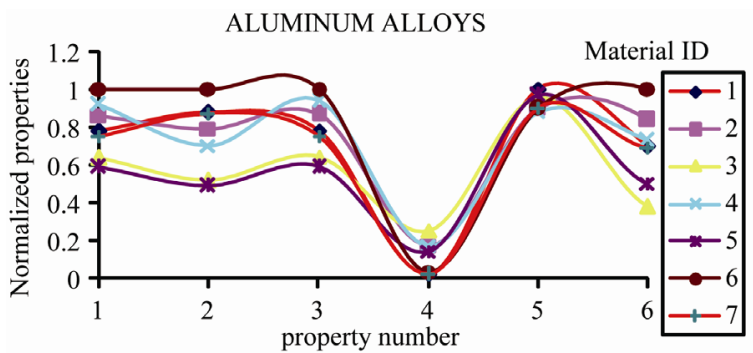

Figure 3. Variation of Normalized Property Values for Various Alloys 
110016, Project ref. No.SR/S3/MERC-81-SERC Engg.

\section{References}

[1] S. M. A. Sapuan, "Concurrent Engineering Design System for Polymeric-Based Composite Automotive Components," PhD Thesis, De Montfort University, Leicester, 1998.

[2] M. Pallot and V. Sandoval, "Concurrent Enterprise in the Era of the Internet and Electronic Commerce," Kluwer Academic Publishers, New York, 1998.

[3] B. Prasad, "Concurrent Engineering Fundamentals, Volume II, Integrated Product and Process Organization," Prentice Hall, New Jersey, 1996.

[4] Y.-E. Nahm and H. Ishikawa, "Integrated Product and Process Modeling for Collaborative Design Environment", Concurrent Engineering: Research and Applications, Vol. 12, No. 1, March 2004, pp. 5-21

[5] M. Yoshioka, "Knowledge Intensive Engineering Framework: KIEF (formerly known as SYS- FUND) Manual," The University of Tokyo, National Center for Science Information Systems, Tokyo, 2000.

[6] O. Tamine' and R. Dillmann, "KaViDo-A Web-Based System for Collaborative Research and Development Processes," Computers in Industry, Vol. 52, No. 1, 2003, pp. 29-45. doi:10.1016/S0166-3615(03)00067-8

[7] A. Balakrishna, R. S. Babu, D. N. Rao, et al., "Integration of CAD/CAM/CAE in Product Development System Using STEP/XML," International Journal on Concurrent Engineering: Research and Application, Vol. 14, No. 2, 2006, pp. 121-128.

[8] P. J. White "Materials Selection: Is the Machine Really Smart Enough?" Proceedings of Society of Plastics Engineers Conference, Hartfort, 1995.
[9] S. M. Sapuan, "A Knowledge-Based System for Materials Selection in Mechanical Engineering Design," Materials and Design, Vol. 22, No. 8, 2001, pp. 687-695. doi:10.1016/S0261-3069(00)00108-4

[10] D.-H. Jee and K.-J. Kang, "A Method for Optimal Material selection Aided With Decision Making Theory," Journal of Materials and Design, Vol. 21, No. 3, June 2000, pp. 199-206. doi:10.1016/S0261-3069(99)00066-7

[11] L. A. Zadeh, "Fuzzy Sets," Information and Control, Vol. 8, No. 3, 1965, pp. 338-353. doi:10.1016/S0019-9958(65)90241-X

[12] R. Knosala and W. Pedrycz, "Evalution of Design Alternatives in Mechanical Engineering," Fuzzy Sets and Systems, Vol. 47, No. 3, 1992, pp. 269-280.

[13] D. Singer, "Fuzzy Algorithms for Planning the MainteNance of large Systems with Insufficient Resources," International Journal of Systems Science, Vol. 21, No. 11, 1990, pp. 2199-2208.

[14] H. Maeda and S. Murakami, "The use of a fuzzy Decision -Making Method in a Large Scale Computer System choice problem," Fuzzy Sets and Systems, Vol. 54, No. 3, 1993, pp. 235-249. doi:10.1016/0165-0114(93)90369-S

[15] D. Cebon and M. F. Ashby, "Computer-Aided Materials Selection for Mechanical Design," Metals and Materials, Vol. 8, No. 1, 1992, pp. 25-30.

[16] D. Cebon and M. F. Ashby, "Picking a Winner: Optimizing Materials Selection," Mater World, Vol. 4, No. 11, 1996, pp. 646-648.

[17] P. Barnard, "Materials Database Save Time," Material Database, News Issue 2, August 1996.

[18] T. W. Liao, “A Fuzzy Multicriteria Decision Making Method for Material Selection," Journal Manufacturing Systems, Vol. 15, No. 1, 1996, pp. 1-12. doi:10.1016/0278-6125(96)84211-7 